OPEN ACCESS

Edited by:

Yan Press,

Ben-Gurion University of the

Negev, Israel

Reviewed by:

Jagadish K. Chhetri,

Capital Medical University, China

Ming Yang,

Sichuan University, China

${ }^{*}$ Correspondence:

Gita Shafiee

gshafiee.endocrine@gmail.com

Ramin Heshmat

rheshmat@tums.ac.ir

Specialty section:

This article was submitted to

Geriatric Medicine

a section of the journal

Frontiers in Medicine

Received: 19 January 2021

Accepted: 16 March 2021

Published: 13 April 2021

Citation:

Shafiee G, Ostovar A, Maleki Birjandi S, Nabipour I, Larijani $B$ and Heshmat R (2021) Development of a Simple and Practical Screening Tool for Detection of Sarcopenia in Older People: The Bushehr Elderly Health Program. Front. Med. 8:655759. doi: 10.3389/fmed.2021.655759

\section{Development of a Simple and Practical Screening Tool for Detection of Sarcopenia in Older People: The Bushehr Elderly Health Program}

\author{
Gita Shafiee ${ }^{1 *}$, Afshin Ostovar ${ }^{2}$, Saba Maleki Birjandi ${ }^{1}$, Iraj Nabipour ${ }^{3}$, Bagher Larijani ${ }^{4}$ \\ and Ramin Heshmat ${ }^{1 *}$
}

${ }^{1}$ Chronic Diseases Research Center, Endocrinology and Metabolism Population Sciences Institute, Tehran University of Medical Sciences, Tehran, Iran, ${ }^{2}$ Osteoporosis Research Center, Institute of Endocrinology and Metabolism, Tehran University of Medical Sciences, Tehran, Iran, ${ }^{3}$ The Persian Gulf Marine Biotechnology Research Center, Bushehr University of Medical Sciences, Bushehr, Iran, ${ }^{4}$ Endocrinology and Metabolism Research Center, Endocrinology and Metabolism Clinical Sciences Institute, Tehran University of Medical Sciences, Tehran, Iran

Background: Sarcopenia is defined by the loss of muscle mass and function with a considerable prevalence which increases morbidity and mortality. We aimed to develop and validate a simple tool for screening of sarcopenia in Iranian older population.

Methods: In this study, we included 2,211 adults aged 60 years or older that participated in the stage II of Bushehr Elderly Health (BEH) program, a population-based prospective cohort study. We defined sarcopenia as reduced skeletal muscle strength plus low muscle mass. The study sample was divided into two parts; development set which were allocated to the development of the model $(n=1,499)$ and validation set $(n=712)$ were allocated to validation of the model.

Results: There were $22.9 \%$ of men and $23.2 \%$ women classified as having sarcopenia based on EWGSOP-2.After selection of variables, the final models named SarSA-Mod (Sarcopenia Scoring Assessment Models) were developed with area to under curves (AUC) of 0.82 (0.79-0.86) and 0.87 (0.84-0.90) in men and women, respectively. The final model included "age," "weight," and "calf circumference" in both sexes. The sensitivity and specificity and positive and negative predictive values for sarcopenia were 84.3, 76.0, 49.8, and $94.5 \%$ for women, $85.4,64.8,40.2$, and $94.2 \%$ for men, respectively. The model performance was tested in the validation set with accuracy 91 and $84 \%$ among women and men, respectively.

Conclusions: Sarcopenia could be detected using SarSA-Mod, as a simple screening test with high accuracy among both sexes. Also, this screening test is valid, feasible, reliable and cost-effective compared to other tools.

Keywords: sarcopenia, screening tool, sensitivity, accuracy, iranian older population 


\section{INTRODUCTION}

Sarcopenia is a geriatric disease, characterized by loss of skeletal muscle mass and muscle function, leading to adverse effects such as physical disability, poor quality of life and increased mortality $(1,2)$. The range of the prevalence of sarcopenia is $5-13 \%$ among older people (3-5). In addition, with the increasing number of the aged people in the world, its prevalence will increase and it is often regarded as a global public health problem $(6,7)$.

Moreover, individuals with sarcopenia are not aware of the disease in the earlier stage but gradually, critical events in physical and functional disability occur (8). Therefore, early detection of individuals at risk of sarcopenia forms the basis for primary prevention in order to reduce the progress of sarcopenia and prevent its severe outcomes $(8,9)$.

European Working Group on Sarcopenia in Older People (EWGSOP) and Asian Working Group for Sarcopenia (AWGS) commonly recommended the use of diagnostic algorithms for sarcopenia and also, they recommended to use of dual-energy X-ray absorptiometry (DXA) and/or bioelectrical impedance analysis (BIA) (3) for diagnosing low muscle mass $(1,10)$. However, these tools and other methods such as magnetic resonance imaging (MRI) and computed tomography (CT) are not recommended as screening tools for the entire population. Besides, they are not available everywhere and need special training. Therefore, screening of all individuals according to EWGSOP or AWGS algorithms with DXA, CT and/or MRI are very costly, time-consuming, and impractical approaches for clinical practice in poor clinical settings (11).

Other screening tools were recently developed to identify older adults at higher risk for sarcopenia. The SARC-F questionnaire is a simple and easy for screening of sarcopenia in older adults (12). However, it has been validated in different population in the world, but the low sensitivity is a problem for a good screening tool. Therefore, for increasing of sensitivity some researchers added simple anthropometric parameters such as calf circumference to the SARC-F. Some studies showed that combination of calf circumference with this questionnaire can improve diagnostic accuracy of SARC-F (13). Another tool for screening of sarcopenia is known as the Mini Sarcopenia Risk Assessment (MSRA) (14) with high sensitivity and specificity compared to SARC-F (15).

Although, there are various screening tools for sarcopenia, there is no consensus on the best tool for all older people in the world and most of these methods have not tested with other ethnic populations. Therefore, in the present study, the aim was to develop a simple, cost-effective, non-invasive model of parameters to identify sarcopenia in order to facilitate sarcopenia screening in clinical setting of Iranian older population. Finally, the accuracy and diagnostic value of this model compared with other screening tools in a community-dwelling older adult population.

\section{MATERIALS AND METHODS}

\section{Study Design and Participants}

The methodology of Bushehr Elderly Health (BEH) program has been previously described elsewhere (16). In summary, the
$\mathrm{BEH}$ program is a prospective population-based cohort study aimed at determining the prevalence and risk factors of noncommunicable diseases (NCD) among a representative sample of urban older population in Bushehr, South Iran. The target population of study was all people aged 60 years and over residing in the city of Bushehr. This population was about 10,000 persons according to District Health Center of Bushehr. We selected participants through a multi-stage, stratified cluster for BEH study. A total 3,000 people participated in the first Phase of this cohort. After 2.5 years, all participants were invited as the second stage of the BEH program for assessing of musculoskeletal disorders and cognitive impairment in these people (Supplementary Figure 1) (17). Until the time of the current study, 2,211 subject entered stage II. All participants signed a written informed consent and the Research Ethics Committee of Bushehr University of Medical Sciences approved the study.

\section{Measurement of Sarcopenic Parameters and Anthropometric Measurements}

Body composition was measured using dual $\mathrm{x}$-ray absorptiometry (DXA, Discovery WI, HologicInc, USA). Appendicular skeletal muscle mass (ASM) for each participant was derived as the sum of upper and lower limb muscle mass and the skeletal muscle mass index (SMI) as ASM $/$ height ${ }^{2}\left(\mathrm{~kg} / \mathrm{m}^{2}\right)$.

Muscle strength was measured by handgrip strength, using a digital dynamometer. The participant seated, elbow at side and $90^{\circ}$ and the hand in a neutral position. The measurement was carried out three times for each hand and maximum grip strength was calculated by taking the highest measurement from both hands (18). Usual walking speed $(\mathrm{m} / \mathrm{s})$ on a 15 feet (4.57meter) course was used as an objective measure of physical performance $(1,19)$. Heights and weights of participants were measured with a fixed stadiometer and a digital scale according to the standard protocol with shoes removed and the participants wearing light clothing. Body mass index (BMI) was calculated as weight $(\mathrm{kg})$ divided by squared height $\left(\mathrm{m}^{2}\right)$. Waist circumference (WC) was measured at a point midway between the iliac crest and the lowest rib in standing position and hip circumference was measured at the most part of the hip, using a flexible tape,. Upper arm circumference was measured at the midpoint between the olecranon process and the acromion of right arm, as well as, forearm circumference was measured from the widest level with the arm hanging freely at the side. Mid-thigh circumference was measured at a midpoint between trochanterion (top of the thigh bone, femur) and tibialelaterale (top of the tibia bone) of right thigh. Calf circumference was measured at the widest level while the participant was standing upright. All measurements were read to the nearest $0.1 \mathrm{~cm}$.

Blood pressure (BP) was measured twice in a seated position after 15 min rest using a standard mercury sphygmomanometer. The average of the two measurements was considered as the participant's blood pressure.

\section{Definition of Sarcopenia}

Sarcopenia was defined as low muscle strength plus reduced skeletal muscle mass based on the criteria set by EWGSOP-2 (2) 
which recommends the use of reference data to determine cut-off points for muscle mass, along with AWGS (10). In a recent study, reference data from a normative Iranian population are available for detecting sarcopenia. Based on these data, the cut-off values for low SMIs were $7.0 \mathrm{~kg} / \mathrm{m} 2$ and $5.4 \mathrm{~kg} / \mathrm{m} 2$ among men and women, respectively (20). The muscle strength were handgrip strength $<26 \mathrm{~kg}$ for men and $<18 \mathrm{~kg}$ for women; while the cutoff value for low physical performance was a usual walking speed $<0.8 \mathrm{~m} / \mathrm{s}$ for both genders $(10,21)$. Using these cut-off points, sarcopenic individuals were identified.

\section{Screening Tools}

The SARC-F questionnaire and SARC-F with calf circumference were used to compare the new tool obtained from the current study. Strength, ambulation, rising from a chair, stair climbing and history of falling are five domains that are assessed. A score of four or more indicates a risk of sarcopenia (12). Another screening tool is SARCF-Calf that comprises five domains of the SARC-F and calf circumference. We used two cut-off points for calf circumference (CC) according to previous studies: (a) CC $\leq 31 \mathrm{~cm}$ for both genders and (b) $\mathrm{CC} \leq 33 \mathrm{~cm}$ for women and $\mathrm{CC} \leq 34 \mathrm{~cm}$ for men. The CC item is scored 0 points when it is above of the cut off and as 10 points if it is below or equals the cut points. A total score $\geq 11$ indicates positive screening for sarcopenia $(2,21)$.

\section{Sarcopenia Scoring Assessment Models (SarSA-Mod)}

In the current study, we developed a statistical model for screening sarcopenia. Proposed model: "Sarcopenia Scoring Assessment Model (SarSA-Mod)" is based on a prediction equation for screening sarcopenia regarding factors effect on this disease in our study population. SarSA-Mod has been built of three variables including age, weight and calf circumference in both genders.

Details on the methods of developing and validation of SarSAMod are explained in the statistical section.

\section{Statistical Analysis}

Differences in between-group characteristics were examined by student's $t$-tests on the whole dataset.

To develop a statistical screening model to identify patients with sarcopenia, "True validation" or "holdout validation" method was used (22). Using random sampling, the study sample was divided into two parts; $67 \%$ of the cases $(n=1,499)$, called "development set," which were allocated to the development of the model as in true validation, and 33\%; one third of the dataset $(n=712)$, named "validation set" were allocated to validation of the model. This method is a cross validation as named the holdout model. After dividing the dataset into two sets as earlier mentioned, analysis for developing the model in the development set begun and all analysis were stratified by sex.

Candidate variables including age, waist circumference, hip circumference, thigh, upper arm circumference, calf circumference and also weight and BMI were selected based on previous studies, cost-effectiveness, feasibility and availability of variables to be measured, and the results of bivariate analysis.

Chi-square test was used to estimate the effect of each variable with the outcome (sarcopenia) as the dependent variable.

Logistic regression analysis was applied in the development of the final model. To choose the best model, we considered the goodness of fit of the models in both genders.

After selecting final model, $B$ coefficient of each variable was used to calculate its index weight. To discriminate the effect of each variable, the values were rounded to the nearest integer and multiplied by 10 , and the final values were used to develop a suitable scoring model.

The ability of the model to separate those with sarcopenia from those without sarcopenia was evaluated using receiver operating characteristic (ROC) curves and the area under the ROC curve. A suitable cut-off point was selected the maximum value of Youden's index with regards to sensitivity and specificity for the model SarSA-Mod $(23,24)$.

Then, sensitivity, specificity, positive and negative predictive values (PPV \& NPV) and the accuracy of the scores were evaluated using the "validation set," which was left aside so far and not engaged in the model development process. In order to select and validate the final criteria for our scoring model, the model was applied to the validation set, using ROC analyses.

All analysis was performed using SPSS (version 16; SPSS Inc., Chicago, IL, USA) and STATA (Release 12. Statistical software. College Station, Texas: STATA Corp LP). $P$-value $<0.05$ was defined as being statistically significant.

\section{RESULTS}

There were $22.9 \%$ of men and $23.2 \%$ women classified as having sarcopenia based on EWGSOP-2. The characteristics of the participants by sex and sarcopenia status are shown in Table 1. Participants with sarcopenia had significantly lower height, weight, BMI, waist and hip circumferences, calf and thigh circumferences, upper arm and forearm circumferences than those with non-sarcopenia in both sexes. Also, those with sarcopenia were older and had smaller handgrip strength, ASM, SMI and usual gait speed compared to those without sarcopenia in both sexes (all $P<0.001$ ). There were no differences in DBP in men and SBP in both sexes irrespective of the presence of sarcopenia.

Table 2 shows the discriminatory performances of the models based on the number of variables. The following predictors were considered including: age, weight, and calf circumference for both sexes. This table presents $B$ coefficient, standard error and index weight of each variable in the final model in both genders. $B$ coefficients were rounded and multiplied by 10 to develop the final models. The formulas of final models were $[(0.2$ $*$ age $($ years $))-(1.7 *$ calf circumference $(\mathrm{cm}))-($ weight $(\mathrm{Kg})$ $+92.56)]$ in women and $[(1.4 *$ age $($ years $))-(1.2 *$ calf circumference $(\mathrm{cm}))-(0.5 *$ weight $(\mathrm{Kg}))-37.42$ ] in men.

The ROC (receiver operating characteristic) curves analyses were performed on the final scoring models for both genders. The 
TABLE 1 | Characteristics of study population.

\begin{tabular}{|c|c|c|c|c|c|c|}
\hline & \multicolumn{3}{|c|}{ Men } & \multicolumn{3}{|c|}{ Women } \\
\hline Age (years) & $68.13 \pm 5.13$ & $74.32 \pm 7.38$ & $<0.001$ & $68.17 \pm 5.66$ & $71.85 \pm 6.95$ & $<0.001$ \\
\hline Height (cm) & $166.81 \pm 6.03$ & $162.85 \pm 6.36$ & $<0.001$ & $152.86 \pm 5.98$ & $150.67 \pm 6.36$ & $<0.001$ \\
\hline Weight (Kg) & $74.74 \pm 11.90$ & $64.00 \pm 9.96$ & $<0.001$ & $70.19 \pm 11.45$ & $54.15 \pm 8.65$ & $<0.001$ \\
\hline Waist Circumference (cm) & $98.56 \pm 10.88$ & $92.40 \pm 10.89$ & $<0.001$ & $103.48 \pm 11.23$ & $90.26 \pm 10.36$ & $<0.001$ \\
\hline Hip circumference (cm) & $100.55 \pm 7.21$ & $95.60 \pm 7.25$ & $<0.001$ & $108.23 \pm 10.18$ & $96.11 \pm 7.83$ & $<0.001$ \\
\hline Thigh circumference (cm) & $50.76 \pm 5.80$ & $47.05 \pm 5.09$ & $<0.001$ & $53.82 \pm 6.74$ & $46.75 \pm 6.17$ & $<0.001$ \\
\hline Calf circumference (cm) & $36.05 \pm 3.43$ & $33.20 \pm 2.83$ & $<0.001$ & $36.67 \pm 3.97$ & $31.63 \pm 2.92$ & $<0.001$ \\
\hline Upper arm circumference(cm) & $30.13 \pm 3.18$ & $27.53 \pm 2.77$ & $<0.001$ & $31.33 \pm 3.51$ & $26.95 \pm 2.99$ & $<0.001$ \\
\hline Appendicular muscle mass $(\mathrm{Kg})$ & $19.36 \pm 2.57$ & $16.24 \pm 1.88$ & $<0.001$ & $14.07 \pm 1.91$ & $11.14 \pm 1.23$ & $<0.001$ \\
\hline $\mathrm{SMI}\left(\mathrm{Kg} / \mathrm{m}^{2}\right)$ & $6.96 \pm 0.80$ & $6.12 \pm 0.57$ & $<0.001$ & $6.03 \pm 0.77$ & $4.90 \pm 0.37$ & $<0.001$ \\
\hline Usual gait speed $(\mathrm{m} / \mathrm{s})$ & $1.00 \pm 0.29$ & $0.81 \pm 0.29$ & $<0.001$ & $0.77 \pm 0.30$ & $0.72 \pm 0.37$ & $<0.001$ \\
\hline
\end{tabular}

Data are shown as mean \pm standard deviation. BMI, body mass index; SBP, systolic blood pressure; DBP, diastolic blood pressure; SMI, skeletal muscle mass index.

TABLE 2 | The results of the multivariate analysis and the development scoring system in development set.

\begin{tabular}{|c|c|c|c|c|}
\hline Variable & B coefficient $(95 \% \mathrm{Cl})$ & Standard error & $P$-value & Score \\
\hline \multicolumn{5}{|l|}{ Men } \\
\hline Age & 0.14 (0.11 to 0.17$)$ & 0.0167 & $<0.001$ & 1.4 \\
\hline Weight & $-0.05(-0.08$ to -0.02$)$ & 0.0155 & 0.002 & -0.5 \\
\hline Calf circumference & $-0.12(-0.22$ to -0.006$)$ & 0.0552 & 0.040 & -1.2 \\
\hline Constant & $-3.742(-7.09$ to -0.39$)$ & 1.7105 & 0.029 & \\
\hline Age & $0.02(-0.01$ to 0.06$)$ & 0.017 & 0.10 & 0.2 \\
\hline Weight & $-0.10(-0.14$ to -0.07$)$ & 0.018 & 0.000 & -1 \\
\hline Calf circumference & $-0.17(-0.27$ to -0.08$)$ & 0.049 & 0.000 & -1.7 \\
\hline Constant & 9.256 (5.620 to 12.862$)$ & 1.840 & 0.000 & \\
\hline Pseudo $R^{2}$ & 0.31 & & & \\
\hline
\end{tabular}

$\mathrm{Cl}$, confidence interval.

full models had values of area under curve (AUC) as 0.82 (95\%CI: 0.79-0.86) for men and 0.87 (95\% CI: 0.84-0.90) for women. Based on the ROC curves analyses, cut-off points of -19.07 and -14.19 were selected for men and women, respectively; as appropriate for the models.

The score of -19.07 correctly classified $69.4 \%$ of men with sarcopenia with a sensitivity of $85.4 \%$ and a specificity of $64.8 \%$ and also, among women the score of -14.19 correctly classified $77.8 \%$ of women with a sensitivity of $84.3 \%$ and specificity of $76.0 \%$.

Next, the models were internally validated using the validation set. The performance of the models did not differ significantly in the development and validation datasets. In the validation sample, the model for men had a sensitivity of $87.6 \%$ and specificity of $62.8 \%$, and correctly classified $69.2 \%$ of cases; and the model for women had sensitivity of $89.1 \%$ and specificity of $77.7 \%$, and correctly classified $80.7 \%$ of patients (Table 3 ).

Table 4 shows the comparison between the screening methods; SarSA-Mod, SARC-F, SARCF-Calf $(31 \mathrm{~cm})$ and SARCF-Calf $(33 / 34 \mathrm{~cm})$ in the total population. The current tool could identify $86 \%$ of men or women with sarcopenia, but the SARC-F questionnaire classified only $42 \%$ of men and $45 \%$ women as screening targets. However, when calf circumference added to SARC-F with both cut-off points, the tools can identify 
TABLE 3 | Performance of the SarSA-Mod in the development and validation samples.

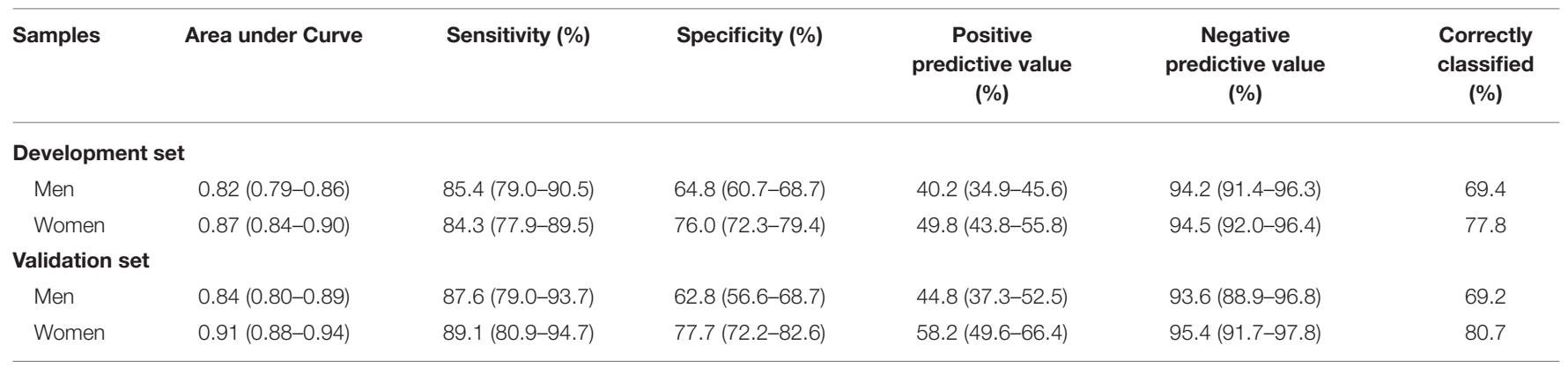

SarSA-Mod, Sarcopenia Scoring Assessment Model.

TABLE 4 | Comparison between the screening methods; SarSA-Mod, SARC-F, SARCF-Calf $(31 \mathrm{~cm})$, and SARCF-Calf $(33 / 34 \mathrm{~cm})$.

\begin{tabular}{|c|c|c|c|c|c|}
\hline & Area under Curve & $\begin{array}{c}\text { Sensitivity } \\
(\%)\end{array}$ & $\begin{array}{c}\text { Specificity } \\
(\%)\end{array}$ & $\begin{array}{c}\text { Positive predictive } \\
\text { value } \\
\text { (\%) }\end{array}$ & $\begin{array}{c}\text { Negative } \\
\text { predictive value } \\
\text { (\%) }\end{array}$ \\
\hline Men & $0.42(0.37-0.47)$ & $13.8(9.8-18.8)$ & $95.9(94.3-97.1)$ & $50.0(37.6-62.4)$ & $78.9(76.2-81.4)$ \\
\hline Women & $0.45(0.41-0.49)$ & $29.6(24.1-35.6)$ & $73.4(70.3-76.4)$ & $25.3(20.4-30.6)$ & $77.6(74.4-80.3)$ \\
\hline Men & $0.49(0.43-0.54)$ & $9.4(6.0-13.7)$ & $98.7(97.6-99.3)$ & $67.7(49.5-82.6)$ & $78.5(75.9-81.0)$ \\
\hline Women & $0.62(0.57-0.66)$ & $28.4(23.0-34.3)$ & $96.1(94.6-97.3)$ & $68.7(59.1-77.5)$ & $81.6(79.0-83.9)$ \\
\hline Total & $0.64(0.62-0.67)$ & $19.1(15.7-22.8)$ & $97.4(96.5-98.1)$ & $68.6(60.2-76.1)$ & $80.0(78.2-81.7)$ \\
\hline \multicolumn{6}{|c|}{ SARCF-Calf $(33 / 34 \mathrm{~cm})$} \\
\hline Men & $0.61(0.56-0.66)$ & $27.2(21.8-33.3)$ & 92.9 (90.9-94.5) & $53.2(44.1-62.1)$ & $81.1(78.4-83.5)$ \\
\hline Women & $0.88(0.86-0.90)$ & $86.1(81.2-90.0)$ & 76.5 (73.5-79.3) & $52.6(47.7-57.5)$ & $94.8(92.8-96.3)$ \\
\hline Total & $0.86(0.84-0.88)$ & $86.1(82.8-89.0)$ & $70.5(68.2-72.6)$ & $46.7(43.4-49.9)$ & 94.4 (93.0-95.6) \\
\hline
\end{tabular}

SarSA-Mod, Sarcopenia Scoring Assessment Model.

sarcopenic patients better than SARC-F alone. SarSA-Mod was superior to SARC-F and SARCF-Calf in terms of AUC, sensitivity and NPV.

The AUC of SarSA-Mod, SARC-F, SARCF-Calf $(31 \mathrm{~cm})$ and SARCF-Calf $(33 / 34 \mathrm{~cm})$ in both sexes of total population are given in Figure 1. The AUCs of SarSA-Mod, SARC-F, SARCFCalf $(31 \mathrm{~cm})$ and SARCF-Calf $(33 / 34 \mathrm{~cm})$ were $0.88,0.53,0.67$ and 0.76 for women, $0.83,0.61,0.64$ and 0.70 among men, respectively $(P<0.001)$.

Also we compared our models with calf circumference alone. The AUCs of calf circumference were 0.20, 0.15, and 0.25 for total population, women and men, respectively (Supplementary Table 1).

\section{DISCUSSION}

In the present study, the sarcopenia screening models for men and women were developed and validated in an Iranian population. Multivariate models were created based on selected variables and good discrimination ability of the models was found with the AUC of 0.82 and 0.87 for men and women, respectively. Based on the ROC curves analyses, the cut-off points of -19.07 and -14.19 were selected for men and women, respectively, and these scores could correctly classify sarcopenic patients with excellent discriminatory power.

To develop SarSA-Mod, important variables associated with sarcopenia or low muscle mass clinically or statistically significant, were examined. Of these factors, the best variables were selected as potential independent parameters of the models in both genders. First, a baseline model was identified according to age and weight which were important factors to develop the model in the previous studies $(25,26)$. Then, the incremental effect of anthropometric parameters to predict sarcopenia was investigated.

Among the anthropometric factors, measurement of calf circumference was simple and feasible and remained in our 
A

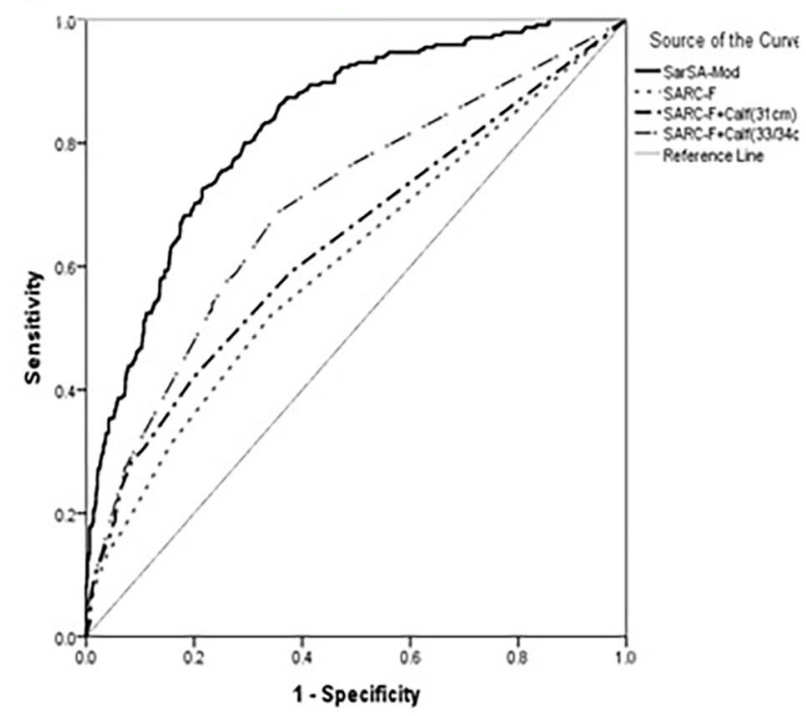

B

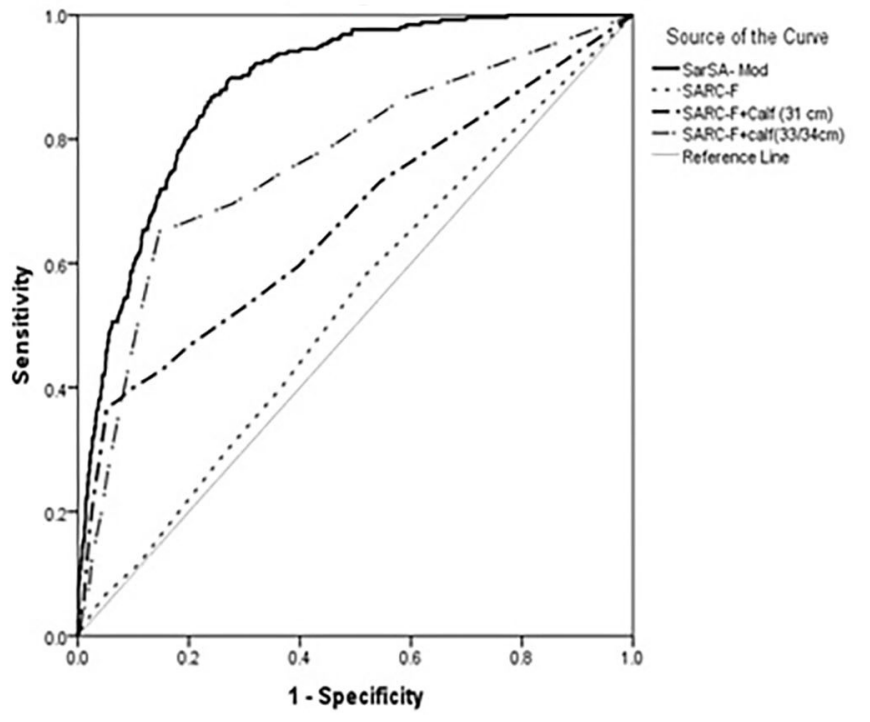

FIGURE 1 | The receiver operating characteristic (ROC) curve for SarSA-Mod (Sarcopenia Scoring Assessment Model), SARC-F, SARCF-Calf (31 cm) and SARCF-Calf $(33 / 34 \mathrm{~cm})$ in the total population in (A) men and (B) women.

multivariate models in both genders. Studies have reported that calf circumference was highly correlated with muscle mass in both genders $(27,28)$. Also, generally, the extremities have a lower fat mass than other body sites (29). So, calf circumference can be used as a replacement indicator of muscle mass for diagnosing sarcopenia.

Therefore, SarSA-Mods were developed based on simple variables including age, weight, and calf circumference to the final model in both sexes. It seems that SarSA-Mods can be easily used in a primary care setting for a screening of sarcopenia in the general population.

In the present study, a scoring system was developed for screening sarcopenia using an index weight of each variable from linear regression analyses. However, several studies attempted for estimation of muscle mass by a variety of variables especially anthropometric parameters (30-32), but few studies developed models with varying degrees of accuracy for sarcopenia which was defined based on muscle mass with muscle function $(12,33)$.

The most common screening tool for sarcopenia is a fivedomain questionnaire, called SARC-F (12). This tool is a simple and quick method and does not require complex measurements. Previous studies showed that the SARC-F could predict adverse outcomes such as hospitalization, poor quality of life, and death $(34,35)$. However, a major weakness of this tool is its low sensitivity which is confirmed by our results and other studies $(13,36)$. The low sensitivity of the SARC-F questionnaire limits its use as a screening tool for sarcopenia because it may miss diagnosing subjects who have sarcopenia (9). For this reason, a research group added calf circumference to the SARC-F to improve diagnostic accuracy and sensitivity of the original SARC-F (37). The findings of this study showed that SARCF-Calf had higher sensitivity and accuracy than SARC-F alone. Similar results from other studies were reported that the addition of calf circumference could increase sensitivity $(13,38)$. In contrast, a study reported that SARCF-Calf had no superiority for sensitivity but improved diagnostic accuracy and specificity (39). In our study, two different cut-off points $(40,41)$ used in the screening of sarcopenia; $31 \mathrm{~cm}$ for both genders, and $33 \mathrm{~cm}$ for women, and $34 \mathrm{~cm}$ for men. Our results indicate that although both SARCFcalf $(31 \mathrm{~cm})$ and SARCF-calf $(33 / 34 \mathrm{~cm})$ improve sensitivity and diagnostic accuracy of SARC-F, there are sensitivity levels of $19.1 \%-38.2 \%$ and accuracy levels of $0.64-0.73$. In line with previous reports $(13,40)$, our findings showed that however, SARCF-Calf has better overall accuracy and sensitivity than SARC-F, but as a screening tool is not perfect.

Some studies exist that have developed the models incorporating the use of the anthropometric equation for muscle mass $(30,31)$. Although, this score has high accuracy for detecting of sarcopenia, these studies attempted to diagnose sarcopenia, according to the recent definitions of sarcopenia, they require the presence of low muscle mass as well as muscle function. So, the present study developed statistical models in both genders for the screening of sarcopenia, which was defined based on muscle mass and muscle function. Also, Ishii and et al. developed a rapid screening test including age, grip strength, and calf circumference for detecting sarcopenia in an Asian population (33). Although this model is very accurate for sarcopenia, the measurement of muscle strength in many medical centers is not feasible due to the lack of dynamometer. We used the variables in our equation can be measured easily and economically in the most clinics even with poor resource.

The discriminative performance of SarSA-Mod was significantly superior to that of SARC-F (AUC $=0.86$ and 
0.57 , respectively, $P<0.001)$. Additionally, SarSA-Mod showed higher sensitivity and NPV than the SARC-F and SARCF-Calf in both genders and total subjects. Therefore, SarSA-Mod as a simple, non-invasive, and feasible tool, with high sensitivity and accuracy is better than SARC-F and SARCF-Calf for the detection of sarcopenia.

The present results have to be interpreted within the context of strengths and potential limitations. First, the studied population was sampled from an urban population; as a result, the study's findings might not be generalizable to the rural population. Second, our models were developed in a cross-sectional cohort and similarly validated in a study set on the same population. There is a need for establishing the external validity of the models in other study populations.

To the best of our knowledge, the current study is the first to develop and validate a sarcopenia screening model for MiddleEast older people. Since SarSA-Mod is easy to calculate with simple variables, it is a useful screening model for sarcopenia in a primary care setting. The scores of SarSA-Mod can be used as an effective screening tool and help in identifying people with sarcopenia for interventions to prevent further adverse events.

\section{DATA AVAILABILITY STATEMENT}

The raw data supporting the conclusions of this article will be made available by the authors, without undue reservation.

\section{ETHICS STATEMENT}

The studies involving human participants were reviewed and approved by Research Ethics Committee of Bushehr

\section{REFERENCES}

1. Cruz-Jentoft AJ, Baeyens JP, Bauer JM, Boirie Y, Cederholm T, Landi F, et al. Sarcopenia: European consensus on definition and diagnosis: report of the European working group on sarcopenia in older people. Age Ageing. (2010) 39:412-23. doi: 10.1093/ageing/afq034

2. Cruz-Jentoft AJ, Bahat G, Bauer J, Boirie Y, Bruyere O, Cederholm T, et al. Sarcopenia: revised European consensus on definition and diagnosis. Age Ageing. (2019) 48:16-31. doi: 10.1093/ageing/afz046

3. Yu S, Umapathysivam K, Visvanathan R. Sarcopenia in older people. Int J Evid Based Healthc. (2014) 12:227-43. doi: 10.1097/XEB.00000000000 00018

4. Castillo EM, Goodman-Gruen D, Kritz-Silverstein D, Morton DJ, Wingard DL, Barrett-Connor E. Sarcopenia in elderly men and women: the rancho bernardo study. Am J Prev Med. (2003) 25:226-31. doi: 10.1016/S0749-3797(03)00197-1

5. Morley JE. Sarcopenia: diagnosis and treatment. J Nutr Health Aging. (2008) 12:452-6. doi: 10.1007/BF02982705

6. Hashemi R, Shafiee G, Motlagh AD, Pasalar P, Esmailzadeh A, Siassi F, et al. Sarcopenia and its associated factors in iranian older individuals: results of SARIR study. Arch Gerontol Geriatr. (2016) 66:18-22. doi: 10.1016/j.archger.2016.04.016

7. Shafiee G, Keshtkar A, Soltani A, Ahadi Z, Larijani B, Heshmat R. Prevalence of sarcopenia in the world: a systematic review and metaanalysis of general population studies. J Diab Met Dis. (2017) 16:21. doi: 10.1186/s40200-017-0302-x
University of Medical Sciences. The patients/participants provided their written informed consent to participate in this study.

\section{AUTHOR CONTRIBUTIONS}

All authors listed have made a substantial, direct and intellectual contribution to the work, and approved it for publication.

\section{FUNDING}

This study was financially supported by a grant from the National Institute for Medical Research Development (Grant No: 940613).

\section{ACKNOWLEDGMENTS}

We would also like to thank all the personnel of the Persian Gulf Tropical Medicine Research Center and all the individuals who took part in the study.

\section{SUPPLEMENTARY MATERIAL}

The Supplementary Material for this article can be found online at: https://www.frontiersin.org/articles/10.3389/fmed. 2021.655759/full\#supplementary-material

Supplementary Figure 1 | The flowchart Bushehr Elderly Health (BEH) Program, phase I.

Supplementary Figure $\mathbf{2}$ | The distributions and histograms of variables of SarSA-Mod in both genders.

Supplementary Table 1 | The area under curve and other characteristics of calf circumference as screening tool for sarcopenia in the whole study population.
8. Visvanathan R, Chapman I. Preventing sarcopaenia in older people. Maturitas. (2010) 66:383-8. doi: 10.1016/j.maturitas.2010.03.020

9. Yu SC, Khow KS, Jadczak AD, Visvanathan R. Clinical screening tools for sarcopenia and its management. Curr Gerontol Geriatr Res. (2016) 2016:5978523. doi: 10.1155/2016/5978523

10. Chen LK, Liu LK, Woo J, Assantachai P, Auyeung TW, Bahyah KS, et al. Sarcopenia in Asia: consensus report of the Asian working group for sarcopenia. J Am Med Dir Assoc. (2014) 15:95-101. doi: 10.1016/j.jamda.2013.11.025

11. Grimes DA, Schulz KF. Uses and abuses of screening tests. Lancet. (2002) 359:881-4. doi: 10.1016/S0140-6736(02)07948-5

12. Malmstrom TK, Morley JE. SARC-F: a simple questionnaire to rapidly diagnose sarcopenia. J Am Med Dir Assoc. (2013) 14:531-2. doi: 10.1016/j.jamda.2013.05.018

13. Yang $\mathrm{M}, \mathrm{Hu} \mathrm{X}, \mathrm{Xie} \mathrm{L}$, Zhang L, Zhou J, Lin J, et al. Screening sarcopenia in community-dwelling older adults: SARC-F vs. SARC-F combined with calf circumference (SARC-CalF). J Am Med Dir Assoc. (2018) 19:277 e1-8. doi: 10.1016/j.jamda.2017.12.016

14. Rossi AP, Micciolo R, Rubele S, Fantin F, Caliari C, Zoico E, et al. Assessing the risk of sarcopenia in the elderly: the mini sarcopenia risk assessment (MSRA) questionnaire. J Nutr Health Aging. (2017) 21:743-9. doi: 10.1007/s12603-017-0921-4

15. Yang $\mathrm{M}, \mathrm{Hu} \mathrm{X}$, Xie L, Zhang L, Zhou J, Lin J, et al. Comparing mini sarcopenia risk assessment with SARC-F for screening sarcopenia in community-dwelling older adults. J Am Med Dir Assoc. (2019) 20:53-7. doi: 10.1016/j.jamda.2018.04.012 
16. Ostovar A, Nabipour I, Larijani B, Heshmat R, Darabi H, Vahdat K, et al. Bushehr elderly health (BEH) programme, phase I (cardiovascular system). BMJ Open. (2015) 5:e009597. doi: 10.1136/bmjopen-2015-009597

17. Shafiee G, Ostovar A, Heshmat R, Darabi H, Sharifi F, Raeisi A, et al. Bushehr elderly health (BEH) programme: study protocol and design of musculoskeletal system and cognitive function (stage II). BMJ Open. (2017) 7:e013606. doi: 10.1136/bmjopen-2016-013606

18. Roberts HC, Denison HJ, Martin HJ, Patel HP, Syddall H, Cooper C, et al. A review of the measurement of grip strength in clinical and epidemiological studies: towards a standardised approach. Age Ageing. (2011) 40:423-9. doi: 10.1093/ageing/afr051

19. Fried LP, Tangen CM, Walston J, Newman AB, Hirsch C, Gottdiener J, et al. Frailty in older adults: evidence for a phenotype. J Gerontol A Biol Sci Med Sci. (2001) 56:M146-56. doi: 10.1093/gerona/56.3.M146

20. Shafiee G, Ostovar A, Heshmat R, Keshtkar AA, Sharifi F, Shadman Z, et al. Appendicular skeletal muscle mass reference values and the peak muscle mass to identify sarcopenia among iranian healthy population. Int J Prev Med. (2018) 9:25. doi: 10.4103/ijpvm.IJPVM_295_17

21. Chen LK, Woo J, Assantachai P, Auyeung TW, Chou MY, Iijima K, et al. Asian working group for sarcopenia: 2019 consensus update on sarcopenia diagnosis and treatment. J Am Med Dir Assoc. (2020) 21:300-7 e2. doi: 10.1016/j.jamda.2019.12.012

22. Picard RR. Cross-Validation of regression models. J Am Stat Asso. (1984) 79:575-83.

23. Perkins NJ, Schisterman EF. The inconsistency of "optimal" cutpoints obtained using two criteria based on the receiver operating characteristic curve. Am J Epidemiol. (2006) 163:670-5. doi: 10.1093/aje/kwj063

24. Fluss R, Faraggi D, Reiser B. Estimation of the youden index and its associated cutoff point. Biom J. (2005) 47:458-72. doi: 10.1002/bimj.200410135

25. Yoshida D, Suzuki T, Shimada H, Park H, Makizako H, Doi T, et al. Using two different algorithms to determine the prevalence of sarcopenia. Geriatr Gerontol Int. (2014) 14(Suppl. 1):46-51. doi: 10.1111/ggi.12210

26. Visvanathan R, Yu S, Field J, Chapman I, Adams R, Wittert G, et al. Appendicular skeletal muscle mass: development and validation of anthropometric prediction equations. J Frailty Aging. (2012) 1:147-51. doi: $10.14283 /$ jfa.2012.23

27. Rolland Y, Lauwers-Cances V, Cournot M, Nourhashemi F, Reynish W, Riviere D, et al. Sarcopenia, calf circumference, and physical function of elderly women: a cross-sectional study. J Am Geriatr Soc. (2003) 51:1120-4. doi: 10.1046/j.1532-5415.2003.51362.x

28. Kawakami R, Murakami H, Sanada K, Tanaka N, Sawada SS, Tabata I, et al. Calf circumference as a surrogate marker of muscle mass for diagnosing sarcopenia in Japanese men and women. Geriatr Gerontol Int. (2015) 15:96976. doi: 10.1111/ggi.12377

29. Tresignie J, Scafoglieri A, Pieter Clarys J, Cattrysse E. Reliability of standard circumferences in domain-related constitutional applications. Am J Hum Biol. (2013) 25:637-42. doi: 10.1002/ajhb.22423

30. Lee RC, Wang Z, Heo M, Ross R, Janssen I, Heymsfield SB. Total-body skeletal muscle mass: development and cross-validation of anthropometric prediction models. Am J Clin Nutr. (2000) 72:796-803. doi: 10.1093/ajcn/72.3.796

31. Kim J, Wang Z, Heymsfield SB, Baumgartner RN, Gallagher D. Totalbody skeletal muscle mass: estimation by a new dual-energy X-ray absorptiometry method. Am J Clin Nutr. (2002) 76:378-83. doi: 10.1093/ajcn/ 76.2.378

32. Pereira PM, da Silva GA, Santos GM, Petroski EL, Geraldes AA. Development and validation of anthropometric equations to estimate appendicular muscle mass in elderly women. Nutr J. (2013) 12:92. doi: 10.1186/1475-289112-92

33. Ishii S, Tanaka T, Shibasaki K, Ouchi Y, Kikutani T, Higashiguchi T, et al. Development of a simple screening test for sarcopenia in older adults. Geriatr Gerontol Int. (2014) 14(Suppl. 1):93-101. doi: 10.1111/ggi.12197

34. Wu TY, Liaw CK, Chen FC, Kuo KL, Chie WC, Yang RS. Sarcopenia screened with SARC-F questionnaire is associated with quality of life and 4-Year mortality. J Am Med Dir Assoc. (2016) 17:1129-35. doi: 10.1016/j.jamda.2016.07.029

35. Malmstrom TK, Miller DK, Simonsick EM, Ferrucci L, Morley JE. SARCF: a symptom score to predict persons with sarcopenia at risk for poor functional outcomes. J Cachexia Sarcopenia Muscle. (2016) 7:28-36. doi: $10.1002 /$ jcsm. 12048

36. Woo J, Leung J, Morley JE. Validating the SARC-F: a suitable community screening tool for sarcopenia? J Am Med Dir Assoc. (2014) 15:630-4. doi: 10.1016/j.jamda.2014.04.021

37. Barbosa-Silva TG, Menezes AM, Bielemann RM, Malmstrom TK, Gonzalez MC, Grupo de Estudos EM, et al. Enhancing SARC-F: improving sarcopenia screening in the clinical practice. J Am Med Dir Assoc. (2016) 17:1136-41. doi: 10.1016/j.jamda.2016.08.004

38. Urzi F, Simunic B, Buzan E. Basis for sarcopenia screening with the SARC-CalF in nursing homes. J Am Med Dir Assoc. (2017) 18:991 e5-10. doi: 10.1016/j.jamda.2017.07.011

39. Bahat G, Oren MM, Yilmaz O, Kilic C, Aydin K, Karan MA. Comparing SARC-F with SARC-CalF to screen sarcopenia in community living older adults. J Nutr Health Aging. (2018) 22:1034-8. doi: 10.1007/s12603-018-1072-y

40. Krzyminska-Siemaszko R, Deskur-Smielecka E, Kaluzniak-Szymanowska A, Lewandowicz M, Wieczorowska-Tobis K. Comparison of diagnostic performance of SARC-F and its two modified versions (SARCCalF and SARC-F+EBM) in community-dwelling older adults from poland. Clin Interv Aging. (2020) 15:583-94. doi: 10.2147/CIA.S2 50508

41. Lim WS, Chew J, Lim JP, Tay L, Hafizah N, Ding YY. Letter to the editor: case for validated instead of standard cut-offs for SARC-CalF. J Nutr Health Aging. (2019) 23:393-5. doi: 10.1007/s12603-019-1177-y

Conflict of Interest: The authors declare that the research was conducted in the absence of any commercial or financial relationships that could be construed as a potential conflict of interest.

Copyright (C) 2021 Shafiee, Ostovar, Maleki Birjandi, Nabipour, Larijani and Heshmat. This is an open-access article distributed under the terms of the Creative Commons Attribution License (CC BY). The use, distribution or reproduction in other forums is permitted, provided the original author(s) and the copyright owner(s) are credited and that the original publication in this journal is cited, in accordance with accepted academic practice. No use, distribution or reproduction is permitted which does not comply with these terms. 